\title{
Erratum: Shot-noise-dominant regime for ellipsoidal nanoparticles in a linearly polarized beam [Phys. Rev. A 95, 053421 (2017)]
}

Changchun Zhong $\mathbb{0}$ and F. Robicheaux

(Received 28 August 2019; published 30 September 2019)

DOI: $10.1103 /$ PhysRevA.100.039903

There was a factor of 2 error in the evaluation of Eqs. (2) and (13) because the polarization of the outgoing photon should have been summed over instead of averaged. Thus, the expressions for the heating rates should be two times larger in Eqs. (9), (10), (19), and (20). The values should be two times larger in columns $6\left(\dot{E}_{R}\right)$ and $7\left(\dot{E}_{T}\right)$ of the tables. The shot-noise $\dot{E}_{T}$ is distributed in the translational degrees of freedom with the ratio $\dot{E}_{x}: \dot{E}_{y}: \dot{E}_{z}=2: 7: 1$. Figure $4-6$ in the main text should be replaced by Figs. 1-3 in this erratum.

The factor 2 error in the heating rate does not affect the cooling trend, thus, will not change the conclusions in the main text.
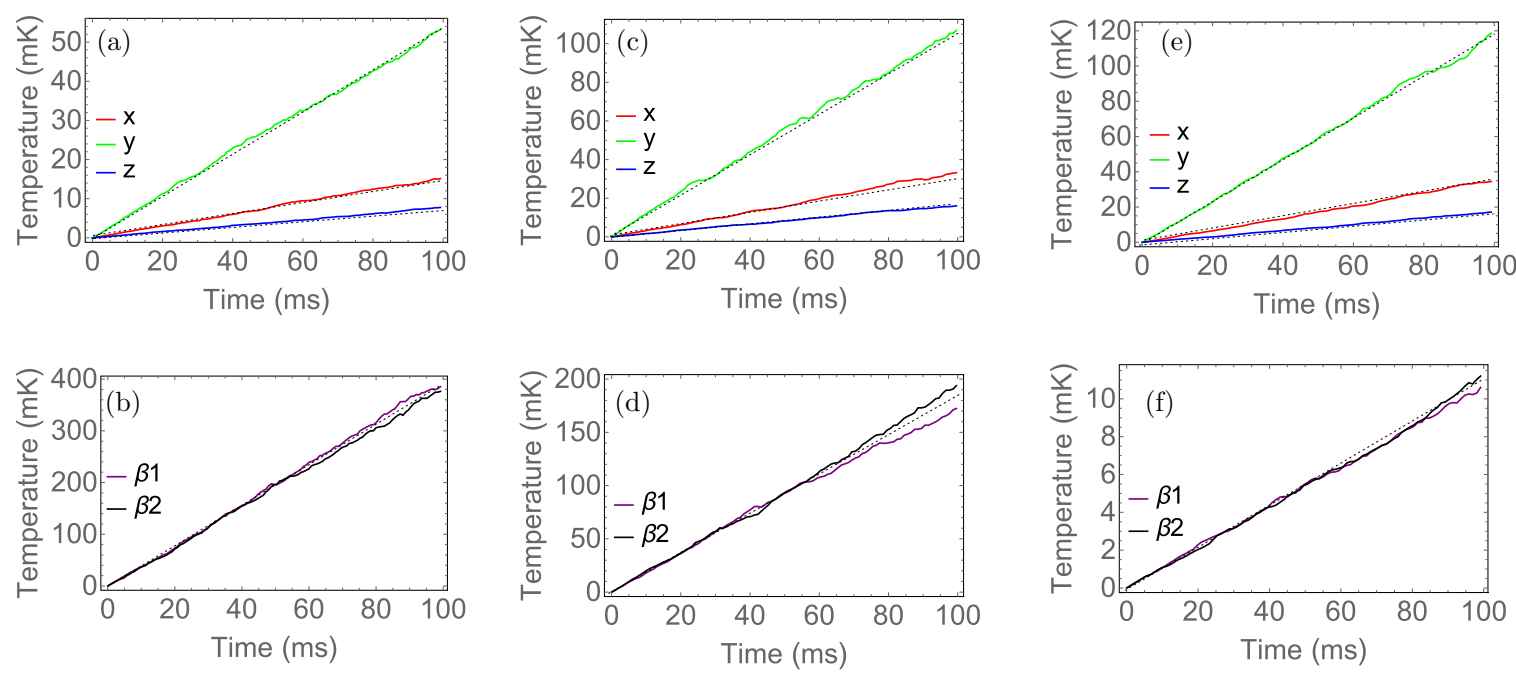

FIG. 1. The classical simulation results of shot-noise heating for nanodiamonds in both the translational and the rotational degrees of freedom. Each curve is averaged over 400 individual reheating trajectories. (a) and (b) are for the nanoparticle with half axes $(a=15$, $b=70 \mathrm{~nm})$, whereas (c) and (d) with half axes $(a=38, b=60 \mathrm{~nm})$, (e) and (f) with half axes $(a=48, b=53 \mathrm{~nm})$. The dashed lines are the heating curves $T=T_{0}+\dot{E} t$ with $T_{0}$ as the initial temperature and $\dot{E}$ as the corresponding heating rate. 

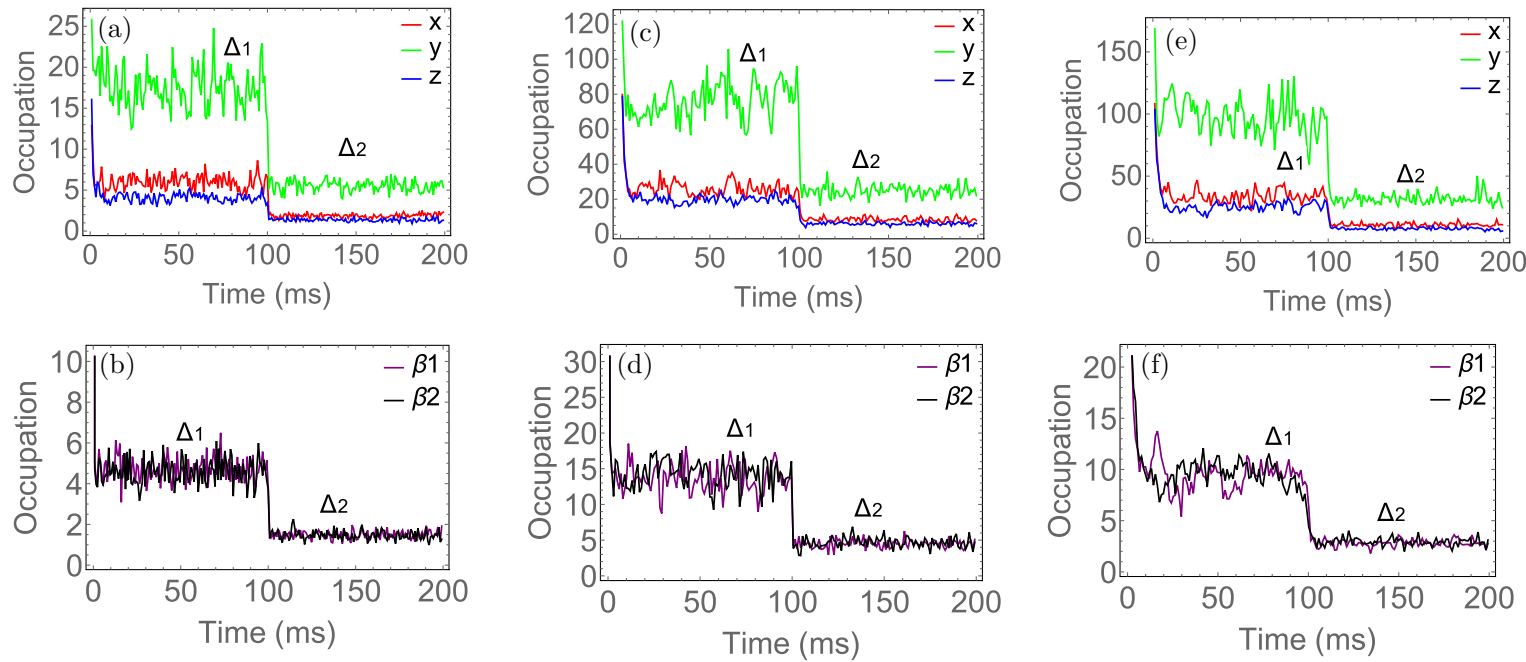

FIG. 2. The parametric feedback cooling for nanodiamonds in all degrees of freedom where each curve shows the time evolution of the average occupation number in the corresponding degree of freedom. Data are collected by averaging 30 cooling trajectories. Calculations are for classical parametric feedback cooling, thus, results for occupation numbers less than ten are suggestive. (a) and (b) depict the translational and rotational coolings, respectively, for a nanoparticle with half axes $(a=15, b=70 \mathrm{~nm})$. The cooling parameter $\Delta_{1}=\left\{\eta_{i}=1.1 \times 10^{11}\right.$, $\left.\zeta_{i}=10^{11} \mathrm{~s} / \mathrm{m}^{2}\right\}$ for $t<100 \mathrm{~ms}$ and $\Delta_{2}=10 \Delta_{1}$ for $t>100 \mathrm{~ms}$. Similarly, (c) and (d) show the cooling for half axes $(a=38, b=60 \mathrm{~nm})$ whereas (e) and (f) for half axes $(a=48, b=53 \mathrm{~nm})$.
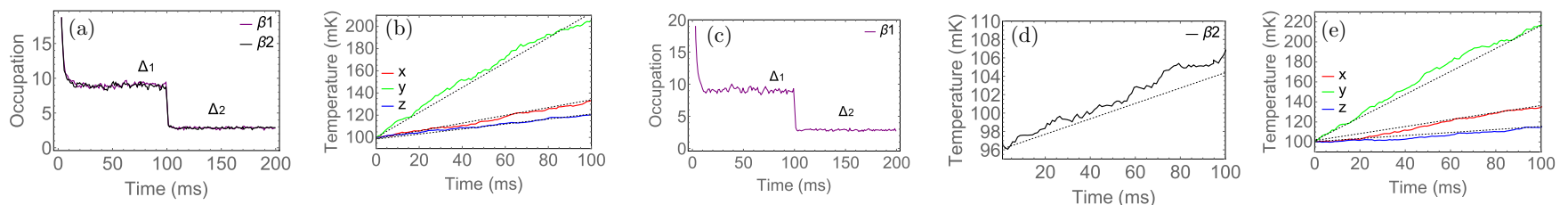

FIG. 3. The parametric feedback cooling in only the rotational degrees of freedom for a nanodiamond $(a=48, b=53 \mathrm{~nm})$. All curves are averaged over 400 trajectories. (a) and (b) show the cooling in both $\beta_{1}$ and $\beta_{2}$ with the cooling parameters $\Delta_{1}=\left\{\eta_{1,2,3}=0, \zeta_{i}=10^{11} \mathrm{~s} / \mathrm{m}^{2}\right\}$. The black and purple lines show the rotational motion gets cooled as we increase the feedback parameters from $\Delta_{1}$ to $\Delta_{2}=10 \Delta_{1}$. The red, green, and blue lines depict that the heating trajectories in translational degrees of freedom. (c)-(e) show the result of cooling in only $\beta_{1}$ with parameters $\Delta_{1}=\left\{\eta_{1,2,3}=0, \zeta_{2}=0, \zeta_{1}=10^{11} \mathrm{~s} / \mathrm{m}^{2}\right\}, \Delta_{2}=10 \Delta_{1}$, and heating in $\beta_{2}$ and $x, y, z$, respectively. In (d), resonance causes more heating in the uncooled $\beta_{2}$ degree of freedom. The dashed lines in (b), (d), and (e) are the heating curves $T=T_{0}+\dot{E} t$ with $T_{0}$ as the initial temperature and $\dot{E}$ as the corresponding heating rate. 